\title{
Editorial: Pet Overpopulation
}

Such a complex issue as that concerning unowned and free roaming pets could not be even sketched in only one issue, so the present issue is an ideal continuation of previous one, concerning companion animals who are not part of a human family. Although we recognize that even the two issues together are far from being able to cover all of the ethical issues related to pet overpopulation management, we hope to have managed nonetheless to provide new insight regarding the ethical concerns raised by some context-specific pet population control situations.

In detail, issue volume 1 , number 2 highlighted mainly the many and complex ethical problems related to the choice of how to deal with "surplus" pets at a large-scale level, highlighting advantages and limits of sheltering and of trap neuter release policies. Also, the effects of adopting a nationwide no-kill policy for healthy, not dangerous dogs, was discussed. Other two scenarios, posing complex large-scale ethical problems were also presented: commercial dog breeding and practices regarding pets in case of non-epidemic emergencies. Also, the first paper of the present issue deals with a situation, which involves not only "surplus" pets, but also family pets, similarly to the one regarding of non-epidemic emergencies. In detail, the first paper of the present issue, authored by Rowan and co-Authors (2020) contributes to the debate (e.g., Robertson, 2008; Crowley et al., 2019) on the effects of cat predation on wildlife, in which cats who have a human family, but are allowed to roam, can play a significant role. However, feral cats play a major role in the problem, and are the target of restrictive measures in several countries, including culling plans. Therefore, Rowan and co-authors (2O2O) provide, not only a balanced analysis of the trends in domestic cat demographics, but also examine the effects of Trap Neuter Return programs for unowned cats.

Also, the rest of the standard papers subsection of the present issue focusses on domestic cats. As with the previous issue, the main focus is on those pets "without a human family", i.e., the ones contributing to the so-called "pet overpopulation" problem, such as feral, stray, colony and shelter cats. Although some issues are common with what happens in dogs, the behavior of cats, who are perceived as less "controllable" than dogs, and to have a different, less cooperative, relationship with humans, can pose specific challenges. Natoli 
and co-authors (2020) explore the national laws concerning unowned freeroaming cat populations in some European countries and discuss some ethical aspects related to the application of the abovementioned regulations, in view of the current knowledge of the social and spatial organization of domestic cats in different situations. Fragoso (2020) highlights some relevant welfare and ethical issues related to the way the problem of cats without a human family is handled and cat (re)homing is conducted by shelters.

In the "perspective papers", the focus returns also on dogs. In the first paper of the sub-section, Robinson and Watkinson (2020) deal with the plea of Spanish hunting dogs (Galgos and Podencos), the abuse they endure at the hands of humans and the work being carried out to help them by ad hoc rescue organizations, which are often faced with ethical dilemmas. In the second, Benedetti and co-authors (2020) explore the ethical implications of the management within shelters of dogs with a history of out of context aggression. In the other two contributions, both Levi (2020) and Friz (2020) draw upon their direct experience with the ethically relevant issues they encountered when dealing with unwanted pets. Levi (2020) reports her experience as the veterinarian in charge of a public animal shelter in Milan, both before and after the enactment of the law enforcing a nation-wide no-kill policy for healthy dogs in shelters, whereas Fritz reports her experience as the manager of a private rescue organization in the South of Italy, where the problem of stray dogs and cats is particularly heavy.

In the non-thematic section, Kollerup Oftedal and Lynning Harfeld explore the relevance of the concept of shame in the dog-human relationship, especially regarding the case of psychiatric service dogs and war veterans suffering from post-traumatic stress disorder.

\section{Acknowledgements}

The guest editors wish to thank all the people who contributed to make this special issue possible (including issues volume 1 , number 2 and volume 2 , number 1) possible: all authors, all reviewers, the Managing Editor, all involved Brill's staff, and JAAER's Editors-in-chief.

\section{Simona Normando}

Università degli Studi di Padova, Padua, Italy

\section{Lucia Selli}

Istituto Zooprofilattico Sperimentale delle Tre Venezie, Legnaro PD, Italy 


\section{References}

Benedetti, R., Malfatti, A., \& Marchegiani, A. (2020). Difficulties in making the ethically correct choice in the management of a case of proven dangerousness of a shelter dog. Journal of Applied Animal Ethics Research, 2:1, this issue.

Crowley, S.L., Cecchetti, M., \& McDonald, R.A. (2019). Hunting behaviour in domestic cats: An exploratory study of risk and responsibility among cat owners. People and Nature, 1:1, pp. 18-30.

Fragoso, S. (2020). Shelter cats: from admission to adoption-ethical and welfare concerns. Journal of Applied Animal Ethics Research, 2:1, this issue.

Friz, D. (2020). Catch neuter release and pet overpopulation management in Southern Italy. Journal of Applied Animal Ethics Research, 2:1, this issue.

Kollerup Oftedal L., \& Lynning Harfeld J. (2020). The relevance of shame in dog-human relationships. Journal of Applied Animal Ethics Research, 2:1, this issue.

Levi, D. (2020). Evolution of stray dog's control in Italy. Journal of Applied Animal Ethics Research, 2:1, this issue.

Natoli, E., Ziegler, N., Dufau, A., \& Teixeira, M.P. (2020). Unowned free-roaming domestic cats: reflection of animal welfare and ethical aspects in animal laws in six European countries. Journal of Applied Animal Ethics Research, 2:1, this issue.

Robertson, S.A. (2008). A review of feral cat control. Journal of Feline Medicine and Surgery, 10, 366-375. doi:10.1016/j.jfms.2007.08.003

Robinson, L., \& Watkinson, J. (2020). Galgos and Podencos in Spain: a rescue's perspective. Journal of Applied Animal Ethics Research, 2:1, this issue.

Rowan, A.N., Kartal, T., \& Hadidian, J. (2020). Cat demographics \& impact on wildlife in the USA, the UK, Australia and New Zealand: facts and values. Journal of Applied Animal Ethics Research, 2:1, this issue. 